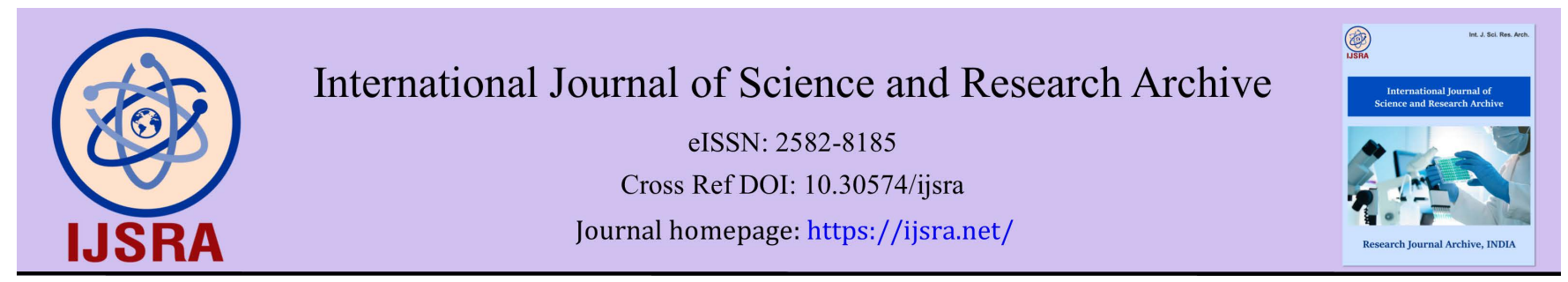

(REVIEW ARTICLE)

\title{
Advances in the pathogenesis and pharmacotherapy of acute stress disorder
}

\author{
Wang Xin-Yue, Wang Tian and Fu Feng-Hua * \\ School of Pharmacy, Yantai University, Yantai 264005, China.
}

International Journal of Science and Research Archive, 2022, 05(01), 016-021

Publication history: Received on 23 December 2021; revised on 24 January 2022; accepted on 26 January 2022

Article DOI: https://doi.org/10.30574/ijsra.2022.5.1.0031

\begin{abstract}
Acute stress disorder (ASD) is a kind of mental disease caused by trauma, which will bring heavy burdens to patients themselves, their family, their work and their lives. Once a person who has suffered a trauma develops post-traumatic stress disorder (PTSD), the quality of life of patients will be overall impaired. It is found that the occurrence of stress disorder is related to the overactivation of hypothalamus pituitary adrenal axis (HPA axis). The most commonly used medications for ASD are recommended medications for PTSD, including serotonin reuptake inhibitors (SRIs), secondgeneration antipsychotics, benzodiazepines, beta blockers, and others. The purpose of this article is to systematically organize and review the possible mechanisms of stress disorder and drugs for treatment of ASD.
\end{abstract}

Keywords: Acute stress disorder; Stress; PTSD; Glucocorticoid

\section{Introduction}

Acute stress disorder (ASD), also known as acute stress reaction (ASR), was proposed in the fourth edition of the Diagnostic and Statistical Manual of Mental Disorders (DSM-IV) in 1994. It usually refers to a mental illness that occurs after the patient has suffered a sharp and severe traumatic event[1]. The standard definition of ASD in the fifth edition of the Diagnostic and Statistical Manual of Mental Disorders (DSM-5) is as follows: Exposure to traumatic events (physical, sexual, or mental); Intrusive symptoms (e.g., always with painful memories of the traumatic event frequently), negative emotions, dissociative symptoms, withdrawal symptoms (e.g., escape to the idea of a traumatic event, memory and feeling, from the traumatic remind from the outside), the arousal symptoms (e.g., sleep problems, distracting); The symptoms mentioned above last from 3 days to 4 weeks and cause significant functional impairment, which is not related to drug use or other treatment $[2,3]$. The duration of symptoms of ASD usually ranges from 3 days to 4 weeks after trauma, and when the duration of symptoms exceeds 4 weeks, it can be defined as post-traumatic stress disorder (PTSD). Symptoms of ASD and PTSD are very similar, but the occurrence time is different[4].

ASD and PTSD are both stress disorders, and their symptoms and conditions can seriously affect individuals' social, occupational, interpersonal lives and physical health. They may also cause significant economic burdens to individuals, families and society. At present, there are different opinions on the relationship between ASD and PTSD. A large number of studies have shown that most people with PTSD do not initially meet the diagnostic criteria for ASD. In contrast, most people with ASD may develop subsequent PTSD[5]. It is thought that there are close relationships between ASD and PTSD[6]. Some papers have reported that ASD is considered as a precursor of PTSD in many trauma survivors. And a lot of ASD patients have been found to eventually develop PTSD[7]. In addition, some researchers believe that the severity of early symptoms in post-traumatic patients is a major predictor of the risk of subsequent PTSD[8]. In conclusion, we believe that ASD is closely related to PTSD. If we can understand the occurrence mechanism of ASD and clarify the relevant treatment drugs for ASD, and then we can make the occurrence and development of ASD under

\footnotetext{
* Corresponding author: Fu Feng-Hua

School of Pharmacy, Yantai University, Yantai 264005, China.
}

Copyright (C) 2022 Author(s) retain the copyright of this article. This article is published under the terms of the Creative Commons Attribution Liscense 4.0. 
control, and the pain and burden of patients will be greatly reduced. Therefore, this paper summarized the advances in the pathogenesis and pharmacotherapy of ASD.

\section{Pathogenesis of ASD}

The effects of traumatic stress on the body are very complex. At present, the specific mechanism of ASD is still not very clear. The type of stressors, intensity and duration of stress will all affect the body. In this condition, most scholars believed that the mechanism of ASD was related to the hypothalamic pituitary adrenal (HPA) axis [9, 10]. In the context of stress, the body's nervous system and endocrine system will change, and hormone levels will also change. The HPA axis is an important part of the neuroendocrine system, participating in the regulation of stress response and playing an important role in the stress system[11]. When the body is exposed to dangerous, unpredictable or uncontrollable environment, negative social evaluation and great mental stress, the HPA axis will be overactivated[12]. When the HPA axis is activated, it will cause the hypothalamus to release large amounts of corticotrophin-releasing hormone (CRH), which acts on the pituitary gland. After that, the pituitary gland can release adrenocorticotropic hormone (ACTH), which can promote the release of glucocorticoid (GCs) from the adrenal cortex. GCs is known as corticosterone in rodents and cortisol in primates. The hippocampus is one of the important target organs of stress, as well as an important brain region for learning and memory[13]. Excessive GCs content will damage the learning and memory process of hippocampus[14]. Synaptic plasticity is recognized as the biological basis of learning and memory activities and is closely related to learning and memory[15]. Under the action of long-term excessive GCs, the morphological structure and function of hippocampal neurons as well as synaptic plasticity will undergo irreversible changes, resulting in various functional disorders[16].Glucocorticoid receptor (GR) and mineralocorticoid receptor (MR) play their roles mainly by binding to two types of receptors. MR is a type I receptor, which exists in the limbic system of the brain, mainly distributed in the hippocampus, parahippocampal gyrus, olfactory cortex and insula. GR belongs to type II receptor and is widely distributed in the whole central nervous system. In general, the affinity of GCs for the two types of receptors is significantly different, with the affinity of GCs for type I receptors being 6-10 times higher than for type II receptors. But under stress condition, type I receptors are saturated because the body releases large amounts of GCs, and large amounts of GCs which bind to type II receptors. Cortisol can shut down the stress response through negative feedback effect. On the one hand, the combination of cortisol and glucocorticoid receptor damages the structure and function of hippocampus; on the other hand, the function and quantity of GR will be down-regulated, which weakens the negative feedback effect on HPA axis, thus forming a vicious cycle[17].

In addition, the excitatory neurotransmitter glutamate (Glu) of the central nervous system, which is involved in a variety of physiological activities in the brain. When the body is stressed, the increase of glutamate increases can result in excitatory toxicity of brain nerves[18]. When glutamate level is pathologically elevated, it can lead to serious damage of neurons.

\section{Pharmacotherapy for ASD}

At present, there is not much evidence about the treatment of ASD, and the current treatment suggestions for ASD are all from the research on the treatment of PTSD[19]. In view of the great similarity between ASD and PTSD, it is believed that the therapeutic drugs of PTSD are also suitable for the treatment of ASD [20].

Therefore, this paper summarizes the drugs related to stress disorder in order to provide some help for clinical research and medication. Treatment methods for ASD can be mainly divided into psychotherapy and drug therapy[21]. Although current studies show that psychological intervention is the first choice after traumatic stress [22]. But psychotherapy takes a long time, has uncertain effects, and may even worsen[23]. Drug therapy aims to ameliorate specific symptoms, such as intrusive memory, anxiety, and obvious avoidance symptoms. Drug therapy plays an important role in the treatment of patients and also contributes to the prevention of stress disorder[24, 25]. The commonly used drugs can be divided into serotonin reuptake inhibitors (SRIs), second-generation antipsychotics, benzodiazepines, $\beta$-blockers and other drugs[6,26].

\subsection{Serotonin reuptake inhibitors (SRIs)}

SRIs are currently the most studied drugs for the treatment of stress disorder with good effects[27]. SRIs are further divided into selective serotonin reuptake inhibitors (SSRIs) and serotonin and norepinephrine reuptake inhibitors (SNRIs). It has been reported in the literature that the first-line drugs for the treatment of stress disorder include three SSRIs, fluoxetine, sertraline and paroxetine, as well as venlafaxine, which is a SNRI[28]. There are two drugs approved by FDA for the treatment of PTSD, namely sertraline and paroxetine, both of which are SSRI[27]. Studies have shown that fluoxetine can improve depression-like mood caused by stress disorder[29]. In addition to SRIs antidepressants, 
the early use of tricyclic antidepressants imipramine in the treatment of ASD can also reduce the symptoms of pediatric burns in clinical studies[30].

\subsection{Second-generation antipsychotics}

The symptoms of stress disorder have some similarities with those of psychosis. For example, patients with stress disorder will have hallucinations or delusions and other symptoms due to the existence of traumatic memories, which will easily be considered as symptoms of mental illness. Therefore, some researchers use antipsychotic drugs to treat PTSD[31]. Second-generation antipsychotics are not first-line drugs for the treatment of stress disorder, but it can be used as a single treatment. At the same time, when psychotherapy and SRIs drugs do not work for patients with anxiety and depression, they can also be combined with SRIs drugs[32]. Physicians should start with a small dose and gradually increase the dose while keeping an eye on the patient. If the patient does not show improvement, the drug cannot be stopped immediately, and the drug should be gradually stopped.

\subsection{Benzodiazepines}

Benzodiazepines are widely used in clinical practice, but there is no evidence of effective treatment for PTSD[27]. 0ne of the reason why some clinicians still use benzodiazepines is that these drugs can control patients' symptoms in time, such as their out-of-control emotions and behaviors[33]. At the same time, some studies show that benzodiazepines are not recommended for the treatment of stress disorder, because they are basically ineffective for stress disorder and even cause serious adverse reactions $[34,35]$. If the drug is not used properly, it will deteriorate patients' traumatic memories and lead to more serious stress disorder symptoms. Relevant data in clinical trials shows that after the use of benzodiazepines, patients' anxiety is controlled in the short term, but the risk of triggering stress disorder symptoms is increased[36]. Another study found that the risk of suicide in patients with stress disorder increased by 3 times after taking benzodiazepines[37]. Therefore, it is recommended that clinicians give strict and careful consideration when prescribing such drugs to patients with stress disorder.

\subsection{Beta blockers}

According to literature reports, the application of beta-blockers such as propranolol can reduce some symptoms of patients with acute stress, such as sympathetic symptoms, for example, tachycardia and sweating, but has no significant effect on the incidence, occurrence and development of stress disorder[38]. However, some studies have found that the use of beta blockers inhibits the development of PTSD in women following cardiac surgery[39]. In addition, propranolol may play a role by blocking traumatic memory consolidation in patients with stress disorder, a conclusion that still needs more research data to support[40]. Taken together, there is not enough evidence on beta-blockers to recommend them as a routine treatment for patients with stress disorder.

\subsection{Other medications}

In addition to beta blockers, GCs, such as hydrocortisone, are recommended as soon as possible after traumatic stress to prevent stress disorder [41]. Studies have shown that hydrocortisone can effectively relieve symptoms related to acute stress in patients with high-risk surgery, severe traumatic injury or acute sepsis[42]. Low concentrations of circulating cortisol are thought to prolong the acute adrenergic response to traumatic events, which may exacerbate the consolidation of traumatic memories, thereby increasing a patient's risk of developing PTSD. Early administration of GCs (e.g., within 6 hours) may prevent this undesirable process by promoting homeostasis in the HPA axis and impelling traumatic memory consolidation and recall[43]. Anti-anxiety drugs are also commonly used in the treatment of ASD and PTSD, which mainly target the anxiety-like emotions of patients and play an important role in the treatment of stress disorder[26].

In addition, some drugs play a role by interfering with traumatic memory, which may improve traumatic memory by interfering with the retrieval and stabilization process of memory reconsolidation. For example, glucocorticoid receptor antagonist, RU38486, may be a potential drug for the treatment of mental disorders related to traumatic memory [44]. In addition, recent studies have found that the nociceptin/orphanin FQ (NOP) system is associated with ASD and PTSD. And NOP system may serve as a viable pathway for the treatment of disease progression. NOP antagonists can serve as drugs for inhibiting the fear response of trauma cuing. It may have beneficial effects on traumatic memory of stress disorder[45].

\section{Summary}

As mentioned above, ASD is closely related to PTSD, and both ASD and PTSD impose a heavy burden on patients. With the quickening pace of modern life, more and more people are facing great pressure. The causes of stress disorder are 
no longer limited to major events such as earthquake and car accident that patients suffer. People will also face the threat of stress disorder due to great pressure from work and life. In view of the great harm and impact of stress disorder, we need to fully understand its mechanism and take intervention measures for its mechanism or symptoms. However, the mechanism of ASD is not very clear at present, which may be closely related to the HPA axis. A more detailed and clear mechanism needs to be further studied by researchers. At the same time, as far as the current situation is concerned, the treatment of ASD has little effect, and there is still a long way to go for the prevention and treatment of ASD.

\section{Conclusion}

The pathogenesis and treatment of ASD still need to be improved. Our systematic review of ASD lays a foundation for the exploration of the treatment and mechanism of ASD, and also points out the direction for the medication of ASD patients.

\section{Compliance with ethical standards}

\section{Acknowledgments}

This work was supported by National Natural Science Foundation of China (No. 81873039).

\section{Disclosure of conflict of interest}

The authors declare no conflict of interests.

\section{References}

[1] Dai W, Liu A, Kaminga A, et al. Prevalence of acute stress disorder among road traffic accident survivors: a metaanalysis [J]. BMC psychiatry. 2018; 18(1): 188.

[2] Cardeña E, Carlson E. Acute stress disorder revisited [J]. Annual review of clinical psychology. 2011; 7(1): 24567.

[3] Regier DA, Kuhl EA, Kupfer DJ. The DSM-5: Classification and criteria changes [J]. World psychiatry. 2013; 12(2): 92-8.

[4] Koopman C, Classen C, Cardeña E, et al. When disaster strikes, acute stress disorder may follow [J]. Journal of traumatic stress. 1995; 8(1): 29-46.

[5] Bryant RA, Creamer M, O'donnell ML, et al. A multisite study of the capacity of acute stress disorder diagnosis to predict posttraumatic stress disorder [J]. Journal of clinical psychiatry. 2008; 69(6): 923-9.

[6] Sijbrandij M, Kleiboer A, Bisson J, et al. Pharmacological prevention of post-traumatic stress disorder and acute stress disorder: a systematic review and meta-analysis [J]. The lancet psychiatry. 2015; 2(5): 413-21.

[7] Bryant R. Acute stress disorder as a predictor of posttraumatic stress disorder: a systematic review [J]. The Journal of clinical psychiatry. 2011; 72(2): 233-9.

[8] Shalev A, Gevonden M, Ratanatharathorn A, et al. Estimating the risk of PTSD in recent trauma survivors: results of the International Consortium to Predict PTSD (ICPP) [J]. World psychiatry: official journal of the World Psychiatric Association (WPA). 2019; 18(1): 77-87.

[9] Jayan D, Deroon-Cassini T, Sauber G, et al. A cluster analytic approach to examining the role of cortisol in the development of post-traumatic stress and dysphoria in adult traumatic injury survivors [J]. Psychoneuroendocrinology. 2021; 89(9): S138-S139.

[10] Gupta S, Guleria R, Szabo Y. MicroRNAs as biomarker and novel therapeutic target for posttraumatic stress disorder in Veterans [J]. Psychiatry research. 2021; 305(11): 42-52.

[11] Bao A, Meynen G, Swaab D. The stress system in depression and neurodegeneration: focus on the human hypothalamus [J]. Brain research reviews. 2008; 57(2): 531-53.

[12] Dickerson S, Kemeny M. Acute stressors and cortisol responses: a theoretical integration and synthesis of laboratory research [J]. Psychological bulletin. 2004; 130(3): 355-91. 
[13] Park H, Lee S, Jung J, et al. Glucocorticoid- and long-term stress-induced aberrant synaptic plasticity are mediated by activation of the glucocorticoid receptor [J]. Archives of pharmacal research. 2015; 38(6): 1204-12.

[14] Sauro M, Jorgensen R, Pedlow C. Stress, glucocorticoids, and memory: a meta-analytic review [J]. Stress (Amsterdam, Netherlands). 2003; 6(4): 235-45.

[15] Connor S, Wang Y. A Place at the Table: LTD as a Mediator of Memory Genesis [J]. The Neuroscientist: a review journal bringing neurobiology, neurology and psychiatry. 2016; 22(4): 359-71.

[16] Blondeau N, Plamondon H, Richelme C, et al. K(ATP) channel openers, adenosine agonists and epileptic preconditioning are stress signals inducing hippocampal neuroprotection [J]. Neuroscience. 2000;100(3): 46574.

[17] Herman J. The Neuroendocrinology of Stress: Glucocorticoid Signaling Mechanisms. 2021;137:105641.

[18] Amin S, El-Aidi A, Ali M, et al. Modification of hippocampal markers of synaptic plasticity by memantine in animal models of acute and repeated restraint stress: implications for memory and behavior [J]. Neuromolecular medicine. 2015; 17(2): 121-36.

[19] Bryant R. The Current Evidence for Acute Stress Disorder [J]. Current psychiatry reports. 2018; 20 (12): 111.

[20] Srivastava A, Opler D. Clinical Use of Prazosin in a Patient With Acute Stress Disorder: A Case Report [J]. Journal of psychiatric practice. $2020 ; 26(3): 246-8$.

[21] Bienvenu T, Dejean C, Jercog D, et al. The advent of fear conditioning as an animal model of post-traumatic stress disorder: Learning from the past to shape the future of PTSD research [J]. Neuron. 2021; 109(15): $2380-97$.

[22] Forbes D, Creamer M, Phelps A, et al. Treating adults with acute stress disorder and post-traumatic stress disorder in general practice: a clinical update [J]. The medical journal of Australia. 2007; 187(2): 120-3.

[23] Chen J, Fortney J, Bergman H, et al. Therapeutic alliance across trauma-focused and non-trauma-focused psychotherapies among veterans with PTSD [J]. Psychological services. 2020; 17(4): 452-60.

[24] Roberts N, Kitchiner N, Kenardy J, et al. Early psychological intervention following recent trauma: A systematic review and meta-analysis [J]. European journal of psychotraumatology. 2019; 10(1): 1695486.

[25] Suliman S, Seedat S, Pingo J, et al. Escitalopram in the prevention of posttraumatic stress disorder: a pilot randomized controlled trial [J]. BMC psychiatry. 2015; 15.

[26] Stewart A, Nguyen M, Poudel M, et al. The failure of anxiolytic therapies in early clinical trials: what needs to be done [J]. Expert opinion on investigational drugs. 2015; 24(4): 543-56.

[27] IPSER J, STEIN D. Evidence-based pharmacotherapy of post-traumatic stress disorder (PTSD) [J]. The international journal of neuropsychopharmacology. 2012; 15(6): 825-40.

[28] Bisson J, Baker A, Dekker W, et al. Evidence-based prescribing for post-traumatic stress disorder [J]. The British journal of psychiatry: the journal of mental science. 2020; 216(3): 125-6.

[29] Chu X, Zhou Y, Hu Z, et al. 24-hour-restraint stress induces long-term depressive-like phenotypes in mice [J]. Scientific reports. 2016; 6: 32935.

[30] Tcheung W, Robert R, Rosenberg L, et al. Early treatment of acute stress disorder in children with major burn injury [J]. Pediatric critical care medicine: a journal of the Society of Critical Care Medicine and the World Federation of Pediatric Intensive and Critical Care Societies. 2005; 6(6): 676-81.

[31] Compean E, Hamner M. Posttraumatic stress disorder with secondary psychotic features (PTSD-SP): Diagnostic and treatment challenges [J]. Progress in neuro-psychopharmacology \& biological psychiatry. 2019; 88: 265-75.

[32] Villarreal G, Hamner MB, Caive JM, et al. Efficacy of Quetiapine Monotherapy in Posttraumatic Stress Disorder: A Randomized, Placebo-Controlled Trial [J]. American journal of psychiatry. 2016; 173(12): 1205-12.

[33] Gilbert M, Dinh La A, Romulo Delapaz N, et al. An Emulation of Randomized Trials of Administrating Benzodiazepines in PTSD Patients for Outcomes of Suicide-Related Events [J]. Journal of clinical medicine. 2020; 9(11): 3492 .

[34] Guina J, Rossetter SR, Derhodes BJ, et al. Benzodiazepines for PTSD: A Systematic Review and Meta-Analysis [J]. Journal of psychiatric practice. 2015; 21(4): 281.

[35] Argolo FC, Cavalcanti-Ribeiro P, Netto LR, et al. Prevention of posttraumatic stress disorder with propranolol: A meta-analytic review [J]. Journal of psychosomatic research. 2015; 79(2): 89-93. 
[36] Von Känel R, Schmid J, Meister-Langraf R, et al. Pharmacotherapy in the Management of Anxiety and Pain During Acute Coronary Syndromes and the Risk of Developing Symptoms of Posttraumatic Stress Disorder [J]. Journal of the American Heart Association. 2021; 10(2): e018762.

[37] Deka R, Bryan C, Lafleur J, et al. Benzodiazepines, Health Care Utilization, and Suicidal Behavior in Veterans With Posttraumatic Stress Disorder [J]. The journal of clinical psychiatry. 2018; 79(6): 17m12038.

[38] Steenen S A, Wijk A V, Van D, et al. Propranolol for the treatment of anxiety disorders: Systematic review and meta-analysis [J]. Journal of psychopharmacology. 2016; 30(2): 128-39.

[39] Tarsitani L, De Santis V, Mistretta M, et al. Treatment with $\beta$-blockers and incidence of post-traumatic stress disorder after cardiac surgery: a prospective observational study [J]. Journal of cardiothoracic and vascular anesthesia. 2012; 26(2): 265-9.

[40] Lonergan MH, Oliverafigueroa LA, Pitman RK, et al. Propranolol's effects on the consolidation and reconsolidation of long-term emotional memory in healthy participants: a meta-analysis [J]. Journal of psychiatry \& neuroscience Jpn. 2013; 38(4): 222-31.

[41] Schelling G, Roozendaal B, Quervain J. Can Posttraumatic Stress Disorder Be Prevented with Glucocorticoids. [J]. Annals of New York academy of sciences. 2010; 1032(1): 158-66.

[42] Birur B, Moore N, Davis L. An Evidence-Based Review of Early Intervention and Prevention of Posttraumatic Stress Disorder [J]. Community mental health journal. 2017; 53(2): 183-201.

[43] Yehuda R. Current status of cortisol findings in post-traumatic stress disorder [J]. Psychiatric clinics of North America. 2002; 25(2): 341-68.

[44] Taubenfeld S, Riceberg J, New A, et al. Preclinical assessment for selectively disrupting a traumatic memory via postretrieval inhibition of glucocorticoid receptors [J]. Biological psychiatry. 2009; 65(3): 249-57.

[45] Taylor R, Jeong I, May M, et al. Fear expression is reduced after acute and repeated nociceptin/orphanin FQ (NOP) receptor antagonism in rats: therapeutic implications for traumatic stress exposure [J]. Psychopharmacology. 2020; 237(10): 2943-58. 